\title{
Effect of immunotherapy on patients with unexplained recurrent spontaneous abortion
}

\author{
Jiao Chen ${ }^{1,2 \#}$, Bowen Liu ${ }^{1,2 \#}$, Ying Zhang ${ }^{1,2 \#}$, Liangfei Ao ${ }^{1,2}$, Zehong Li $^{1,2}$, Bing $\mathbf{Q u}^{1,2}$, Xueyao Li $^{1,2}$, Jing Yang ${ }^{1,2}$ \\ ${ }^{1}$ Reproductive Medical Center, Renmin Hospital of Wuhan University, Wuhan, China; ${ }^{2}$ Hubei Clinical Research Center for Assisted Reproductive \\ and Embryonic Development, Wuhan, China \\ Contributions: (I) Conception and design: J Chen, B Liu, Y Zhang, J Yang; (II) Administrative support: J Yang; (III) Provision of study materials or \\ patients: All authors; (IV) Collection and assembly of data: All authors; (V) Data analysis and interpretation: J Chen, B Liu, Y Zhang; (VI) Manuscript \\ writing: All authors; (VII) Final approval of manuscript: All authors. \\ \#These authors contributed equally to this work. \\ Correspondence to: Jing Yang. Reproductive Medical Center, Renmin Hospital of Wuhan University, Wuhan, China. Email: dryangqing@hotmail.com.
}

Background: At present, it is generally believed that immune factors account for $60 \%$ of unexplained recurrent spontaneous abortion (URSA). The treatments used for URSA depend on immunomodulation for their effects, and paternal immunization, intravenous immunoglobulin, and the use of growth factors such as granulocyte-colony stimulating factor (filgrastim) have been shown to have a beneficial effect on patients with a poor prognosis. However, these treatment schemes and effects remain controversial. This study aimed to evaluate the effect of immunotherapy using lymphocyte active immunotherapy (LAI) on patients with URSA, and to provide evidences for the clinical effect of this treatment.

Methods: The detailed data of total 619 patients with URSA were collected and analyzed, of which 465 patients (LAI group) with immunotherapy and 154 patients (control group) without immunotherapy.

Results: After $77.6 \%$ of all the patients in LAI group received the immunotherapy, the maternal blocking antibody (BA) was changed from negative to positive. The conversion rate of maternal BA was increased as the increase of active immunization ( $>4$ times, $\mathrm{P}<0.05$ ). The pregnancy rate of LAI Group was higher than that of the control group $(\mathrm{P}<0.05)$, and there were significant differences of live rate and abortion rate $(\mathrm{P}<0.05)$. In addition, compared with the natural pregnancy, the live rate was higher, and the abortion rate was lower in in vitro fertilization (IVF) patients after active immunization, although the difference was not significant $(\mathrm{P}>0.05)$.

Conclusions: After lymphocyte immunotherapy, most of the patients with unexplained recurrent spontaneous abortion had the positive BA instead of negative BA. Whether the BA was converted or not, the pregnancy rate and live rate were increased, and the abortion rate was decreased after immunotherapy. Therefore, active immunotherapy could improve the pregnancy outcome of the patients with unexplained recurrent abortion.

Keywords: Immunotherapy; blocking antibody; recurrent spontaneous abortion; in vitro fertilization (IVF)

Submitted Nov 03, 2019. Accepted for publication May 28, 2020.

doi: 10.21037/apm-19-440b

View this article at: http://dx.doi.org/10.21037/apm-19-440b

\section{Introduction}

Recurrent spontaneous abortion (RSA) refers to spontaneous abortion that occurs for more than three times continuously between the patient and the same partner (1). Other studies showed that more than $60 \%$ of the women, who have two consecutive spontaneous abortions, were more likely to have spontaneous abortion in a second pregnancy. Therefore, two consecutive spontaneous abortions could be called recurrent abortion $(2,3)$. At present, it is generally believed that immune factors account for $60 \%$ of unexplained recurrent spontaneous abortion (URSA), of which alloimmune recurrent abortion is more 


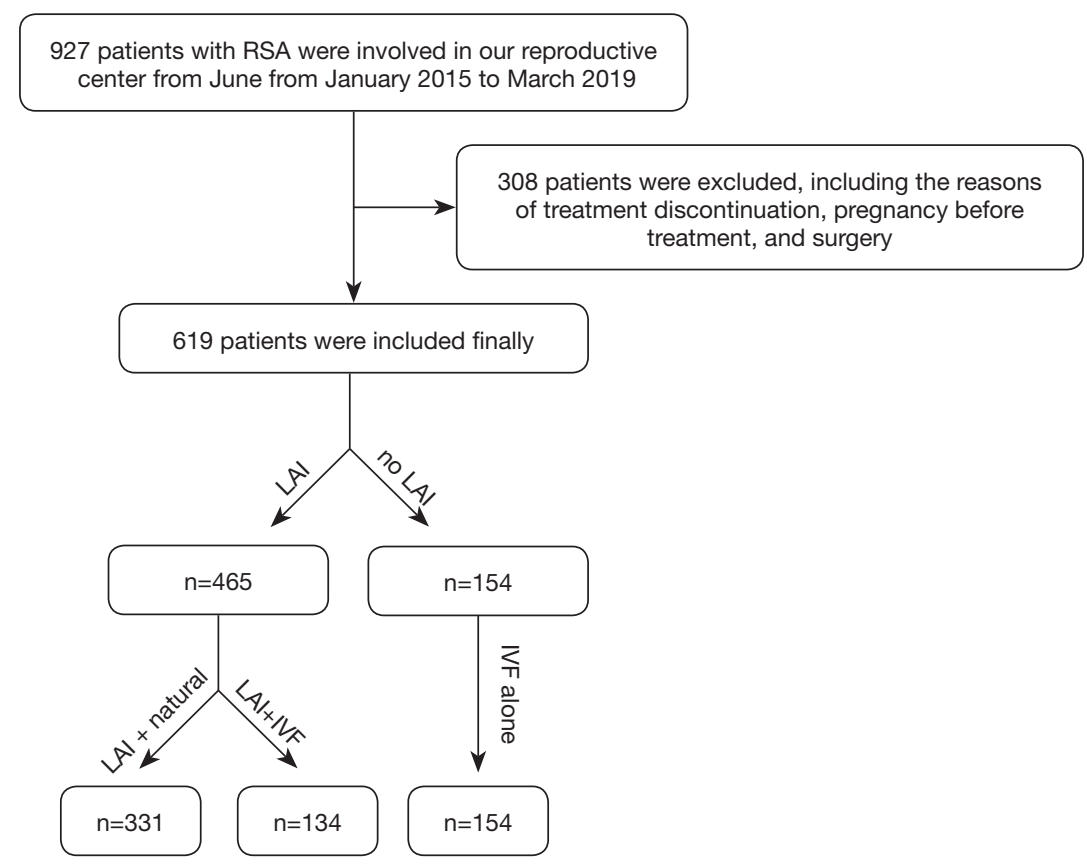

Figure 1 Study design. RSA, recurrent spontaneous abortion; LAI, lymphocyte active immunotherapy; IVF, in vitro fertilization.

common, mainly due to the abnormal recognition of fetal paternal antigen by the mother (4), resulting in the lack of maternal blocking antibody (BA), other cellular and humoral immune abnormalities, embryo suffering from abnormal immune system attacking (5). All treatments used for URSA depend on immunomodulation for their effects, and paternal immunization, intravenous immunoglobulin (IVIg), and the use of growth factors such as granulocytecolony stimulating factor (G-CSF) (filgrastim) have been shown to have a beneficial effect in patients with a poor prognosis and when used appropriately, however, these treatment schemes and effects remain controversial (3). The purpose of this study is to explore the therapeutic effect of BA and pregnancy outcome in RSA patients after paternal lymphocyte active immunotherapy (LAI), so as to provide evidence for the clinical effect of this treatment.

\section{Methods}

\section{General information}

From January 2015 to March 2019, 619 patients with RSA who received medical service in our hospital were involved. Inclusion criteria were as follows: (I) the patients were married; (II) there were more than 2 clinical abortions during early pregnancy; (III) maternal BA were all negative;
(IV) the chromosome of the couples were normal; (V) no abnormal reproductive tract anatomy by hysteroscopy or ultrasound examination. Exclusion criteria: (I) abnormal immune function or diseases affecting pregnancy; (II) reproductive tract infection and uterine malformation; (III) endocrine hormone disorder. All patients were divided into study group (LAI group) and control group. A total of 465 patients in the study group were $31.62 \pm 4.26$ years old, and 154 patients in the control group were $31.49 \pm 4.82$ years old on average. There was also no significant difference between two groups for general information $(\mathrm{P}>0.05)$ (Figure 1). The study was conducted in accordance with the Declaration of Helsinki. The LAI involved in this study has been approved by the Medical Ethics Committee of Renmin Hospital of Wuhan University (No. 20130506). All patients in this study have signed the informed consent at enrollment.

\section{Active immunization in LAI group}

Twenty $\mathrm{mL}$ Ficoll lymph separation solution was added into $50 \mathrm{~mL}$ centrifuge tube, then $20 \mathrm{~mL}$ anticoagulant venous blood was taken from the patient's husband [if the patient's husband is suffering from the acquired immune deficiency syndrome (AIDS), etc., blood can be taken from healthy volunteers], and slowly spread on the liquid surface of lymph 
Table 1 The characteristics of the included population

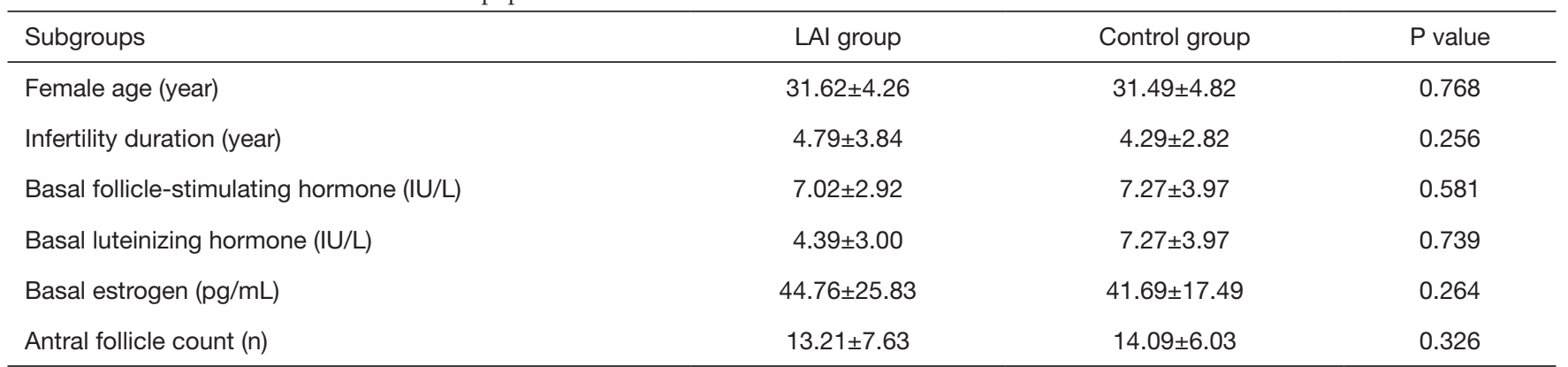

LAl, lymphocyte active immunotherapy.

separation along the tube wall; centrifuged at 3,500 rpm for 20 minutes to get the monocyte layer; centrifuged at $1,500 \mathrm{rpm}$ for 10 minutes to discard the supernatant; the number of cells was adjusted to $1 \times 10^{8} / \mathrm{mL}$ in sterile saline after washed twice. The patients were inoculated at $6-$ 8 points on the medial side of the forearm once every 2 weeks, four times for a course. After that, the patients returned to the hospital to detect the BA. If the antibody was positive, patients might become pregnant [through natural or in vitro fertilization (IVF)]. If pregnancy, immunotherapy was still continued once every month for 4 times. Control group: IVF for pregnancy after serial examinations.

\section{BA test}

Enzyme linked immunosorbent assay instrument was used to detect BA in the blood of patients, and optical density was measured (cut off $=0.2,>0.2$ as positive, otherwise negative).

\section{Observation indexes}

(I) the changes of the patients before and after the treatment with BA; (II) the pregnancy rate, live rate and abortion rate of the patients; (III) IVF and natural pregnancy rate after the treatment with BA.

\section{Statistical analysis}

The data of this study were sorted out by SPSS 21.0 software. The count data were expressed by $\mathrm{N}$, and tested by Chi-square test between two groups; the measurement data were expressed by $\bar{x} \pm S$, and tested by $T$ test between two groups; $\mathrm{P}<0.05$ was considered statistically significant.

\section{Results}

\section{General information}

Differences in female age, infertility duration, basal folliclestimulating hormone/luteinizing hormone/estrogen and antral follicle count between LAI group and control group were not statistically significant $(\mathrm{P}>0.05)$ (Table 1). For IVF patients, differences in controlled ovarian stimulation parameters, including days of gonadotropins, total gonadotropins, estrogen/progesterone value in trigger day, and the endometrial thickness on the day of embryo transfer between LAI group and control group were also not statistically significant $(\mathrm{P}>0.05)$ (Table 2$)$.

\section{Effect of active immunization on maternal BA}

After $77.6 \%$ (361/465) of all the patients in LAI group received the immunotherapy, the maternal BA was changed from negative to positive. The conversion rate of maternal $\mathrm{BA}$ was increased as the increase of active immunization $(\mathrm{P}<0.0001)$ (Table 3).

\section{Comparison in pregnancy rate, live rate and abortion rate between LIT group and control group}

The pregnancy rate of IVF in LAI group was higher than that of the control group ( $97.0 \%$ vs. $53.2 \%, \mathrm{P}<0.0001)$, and the pregnancy rate of IVF was higher than that of natural pregnancy in LIT group $(97.0 \%$ vs. $61.6 \%, \mathrm{P}<0.0001)$. There were significant differences on live rate and abortion rate between LIT group and control group $(80.8 \%$ vs. 
Table 2 Controlled ovarian stimulation parameters of IVF patients

\begin{tabular}{|c|c|c|c|}
\hline Subgroups & LAI group & Control group & $P$ value \\
\hline Total gonadotropins $(U)$ & $2,191.11 \pm 762.12$ & $2,279.52 \pm 778.85$ & 0.371 \\
\hline Trigger day estrogen $(\mathrm{pg} / \mathrm{mL})$ & $4,713.88 \pm 14,734.00$ & $3,977.66 \pm 2,368.25$ & 0.543 \\
\hline Trigger day progesterone $(\mathrm{ng} / \mathrm{mL})$ & $0.98 \pm 0.49$ & $1.07 \pm 0.51$ & 0.836 \\
\hline
\end{tabular}

IVF, in vitro fertilization; LAI, lymphocyte active immunotherapy.

Table 3 Conversion rate of block antibody in study group

\begin{tabular}{|c|c|c|c|}
\hline Subgroups & $<4$ times & $4-6$ times & $>6$ times \\
\hline
\end{tabular}

Table 4 Comparison in pregnancy rate, live rate and abortion rate between LAI group and control group

\begin{tabular}{|c|c|c|c|c|}
\hline Subgroups & Treatment & Pregnancy & Live births & Abortions \\
\hline \multirow[t]{2}{*}{ LAl group } & IVF & 130/134 (97.0\%) & $105(80.8 \%)$ & $11(8.5 \%)$ \\
\hline & Natural pregnancy & 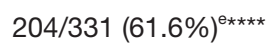 & 150 (73.5\%) & 18 (8.8\%) \\
\hline
\end{tabular}

${ }^{a}$, LAl for less than 4 vs. $4-6$ times; ${ }^{d}$, Control group vs. LAl group with IVF; ${ }^{e}$, IVF vs. natural pregnancy in LAl group; ${ }^{*} \mathrm{P}<0.05 ;{ }^{* \star \star *} \mathrm{P}<0.0001$.

LAI, lymphocyte active immunotherapy; IVF, in vitro fertilization.

$53.7 \%, \mathrm{P}<0.0001 ; 8.5 \%$ vs. $18.3 \%, \mathrm{P}<0.05)$. In addition, compared with natural pregnancy, the live rate was higher, and the abortion rate was lower in IVF patients after active immunization, although the difference was not significant (80.8\% vs. $73.5 \%, \mathrm{P}>0.05 ; 8.5 \%$ vs. $8.8 \%, \mathrm{P}>0.05$ ) (Table 4 ).

\section{Effect of BA conversion on pregnancy outcome in LAI group}

There were significant differences on live rate between positive BA patients and negative BA patients with IVF (85.4\% vs. $63.0 \%, \mathrm{P}<0.01)$, and There were significant differences on live rate between positive BA patients and negative $\mathrm{BA}$ patients with natural pregnancy $(78.4 \%$ vs. $60.7 \%, \mathrm{P}<0.05)$. Although abortion rate of positive BA patients with IVF or natural pregnancy were also decreased, the difference was not significant $(7.8 \%$ vs. $11.1 \%, \mathrm{P}>0.05$; $8.1 \%$ vs. $10.7 \%, \mathrm{P}>0.05$ ) (Table 5 ).

\section{Discussion}

There are many reasons for RSA, including gene, anatomy, endocrine, immune function and so on. It has been shown that the occurrence of URSA is closely related to the immune imbalance (6). The immune system of the normal pregnant mother does not reject the fetus, as a foreign substance (7). Successful pregnancy is closely related to the maternal immune system, as well as the specific antibodies produced by the mother. The BA, as an important one of specific antibodies, can maintain the normal growth and development of embryo. In the early stage of pregnancy, if BA is deficient due to abnormal mother's immune system, the embryo will be repelled and early abortion occurred (8). In LAI, through the transplantation of lymphocytes from the husband into the patient's body, the patient's immune system is stimulated to promote the production of BA (9), enabling the patient to achieve a successful pregnancy. If the 
Table 5 Effect of BA conversion on pregnancy outcome in LAI group

\begin{tabular}{|c|c|c|}
\hline Subgroups & $\mathrm{BA}(+)$ & $\mathrm{BA}(-)$ \\
\hline LAI + natural pregnancy live rate & $78.4(116 / 148)^{9 *}$ & $60.7(34 / 56)$ \\
\hline LAI + IVF abortion rate & $7.8(8 / 103)$ & $11.1(3 / 27)$ \\
\hline LAI + natural pregnancy abortion rate & $8.1(12 / 148)$ & $10.7(6 / 56)$ \\
\hline
\end{tabular}

patient's husband is suffering from AIDS, etc., blood can be taken from healthy volunteers, which greatly avoids the occurrence of infectious diseases.

As early as 1981, Taylor et al. proposed that immunotherapy could be effect on patients with RSA and negative BA (10). Chen et al. also showed that active immunotherapy could significantly improve the pregnancy outcome of patients with RSA (11). However, it was suspected that immunotherapy could not improve the pregnancy outcome of patients with RSA (12). Therefore, the effect of immunotherapy has been controversial for many years. Recent studies suggested that BA played an important role on the treatment of unexplained recurrent abortion (13-15). On the one hand, the method can improve the level of BA in the body and increase the pregnancy rate; on the other hand, it can induce the maternal immune tolerance to the fetus, and regulate the immune imbalance between the mother and the fetus to avoid abortion.

Our data showed the maternal BA was changed from negative to positive, after $77.6 \%$ of all the patients in LAI group received the lymphocytes immunotherapy from the husband. The conversion rate of maternal BA was increased as the increase of active immunization $(>4$ times, $\mathrm{P}<0.05)$. The pregnancy rate of LAI Group was higher than that of the control group $(\mathrm{P}<0.05)$, and there were significant differences on live rate and abortion rate $(\mathrm{P}<0.05)$. In addition, compared with the natural pregnancy, the live rate was higher, and the abortion rate was lower in IVF patients after active immunization, although the difference was not significant $(\mathrm{P}>0.05)$. It was suggested that active immunotherapy could convert BA and improve the pregnancy outcome significantly. However, there are also some problems. For example, those patients who cannot achieve the natural pregnancy within 3-6 months after active immunization, still need to look for the help of IVF. There are also patients who still have a negative BA after a course of treatment, may need the second or even the third course of treatment. Along with the time, the patients want to receive IVF urgently, instead of active immunization. In addition, there are also some people, who are pregnant successfully but miscarry again after treatment, which weakens confidence of patients, and also confuses doctors. It is also proposed in the literature that the effect of different treatment methods and doses is also quite different, so the standardized treatment in clinical needs to be made $(16,17)$.

In conclusion, the serum BA can be routinely detected or the patients with RSA. If people have a negative BA, lymphocyte active immunization will be recommended. If the BA is converted to positive after treatment, natural pregnancy will be recommended. If the patient has an urgent desire for pregnancy, IVF can be used immediately. However, at present, the effect of active immunotherapy on RSA still needs to be studied in larger samples, and its mechanism still needs to be further explored (12).

\section{Acknowledgments}

Funding: This work was supported by grants from the National Natural Science Foundation of China (Award Number: 81571513 \& 81771618 , Recipient: Jing YANG), and the Independent Scientific Research Foundation of Wuhan University (Award Number: 413000266, Recipient: Chen Jiao).

\section{Footnote}

Data Sharing Statement: Available at http://dx.doi. org/10.21037/apm-19-440b

Conflicts of Interest: All authors have completed the ICMJE uniform disclosure form (available at http://dx.doi. 
org/10.21037/apm-19-440b). The authors have no conflicts of interest to declare.

Ethical Statement: The authors are accountable for all aspects of the work in ensuring that questions related to the accuracy or integrity of any part of the work are appropriately investigated and resolved. The study was conducted in accordance with the Declaration of Helsinki. The LAI involved in this study has been approved by the Medical Ethics Committee of Renmin Hospital of Wuhan University (No. 20130506). All patients in this study have signed the informed consent at enrollment.

Open Access Statement: This is an Open Access article distributed in accordance with the Creative Commons Attribution-NonCommercial-NoDerivs 4.0 International License (CC BY-NC-ND 4.0), which permits the noncommercial replication and distribution of the article with the strict proviso that no changes or edits are made and the original work is properly cited (including links to both the formal publication through the relevant DOI and the license). See: https://creativecommons.org/licenses/by-nc-nd/4.0/.

\section{References}

1. Azizi R, Soltani-Zangbar MS, Sheikhansari G, et al. Metabolic syndrome mediates inflammatory and oxidative stress responses in patients with recurrent pregnancy loss. J Reprod Immunol 2019;133:18-26.

2. An HJ, Kim JH, Ahn EH, et al. 3'-UTR Polymorphisms in the Vascular Endothelial Growth Factor Gene (VEGF) Contribute to Susceptibility to Recurrent Pregnancy Loss (RPL). Int J Mol Sci 2019;20:3319.

3. Carp H. Immunotherapy for recurrent pregnancy loss. Best Pract Res Clin Obstet Gynaecol 2019;60:77-86.

4. Krieg S, Westphal L. Immune Function and Recurrent Pregnancy Loss. Semin Reprod Med 2015;33:305-12.

5. Gao HY, Tao EX, Wang Y, et al. Immunomodulatory and clinical effects of the "tiaomian III decoction" in patients with blood blocking antibody deficiency and recurrent spontaneous abortion. Genet Mol Res 2015;14:3421-5.

6. Krog MC, Kolte AM, Husby K, et al. Recurrent pregnancy loss. Ugeskr Laeger 2017;179:V11160834.

7. Bonney EA. Alternative theories: Pregnancy and immune tolerance. J Reprod Immunol 2017;123:65-71.

8. Meuleman T, Lashley LE, Dekkers OM, et al. HLA associations and HLA sharing in recurrent miscarriage: A systematic review and meta-analysis. Hum Immunol 2015;76:362-73.

9. Raspollini MR, Rossi Degl'Innocenti D, Castiglione F, et al. Could gamma/delta T cells influence the mother's rejection in recurrent aborted embryos? J Obstet Gynaecol 2007;27:165-7.

10. Taylor C, Faulk WP. Prevention of recurrent abortion with leucocyte transfusions. Lancet 1981;2:68-70.

11. Chen JL, Yang JM, Huang YZ, et al. Clinical observation of lymphocyte active immunotherapy in 380 patients with unexplained recurrent spontaneous abortion. Int Immunopharmacol 2016;40:347-50.

12. Achilli C, Duran-Retamal M, Saab W, et al. The role of immunotherapy in in vitro fertilization and recurrent pregnancy loss: a systematic review and meta-analysis. Fertil Steril 2018;110:1089-100.

13. Liu Z, Xu H, Kang X, et al. Allogenic Lymphocyte Immunotherapy for Unexplained Recurrent Spontaneous Abortion: A Meta-Analysis. Am J Reprod Immunol 2016;76:443-53.

14. Liu M, Zhen X, Song H, et al. Low-dose lymphocyte immunotherapy rebalances the peripheral blood Th1/ Th2/Treg paradigm in patients with unexplained recurrent miscarriage. Reprod Biol Endocrinol 2017;15:95.

15. Cavalcante MB, Sarno M, Araujo Junior E, et al. Lymphocyte immunotherapy in the treatment of recurrent miscarriage: systematic review and meta-analysis. Arch Gynecol Obstet 2017;295:511-8.

16. Cavalcante MB, Sarno M, Araujo Júnior E, et al. Lymphocyte immunotherapy in the treatment of recurrent miscarriage: systematic review and meta-analysis. Arch Gynecol Obstet 2017;295:511-8.

17. Wilczyński JR, Radwan P, Tchorzewski H, et al. Immunotherapy of patients with recurrent spontaneous miscarriage and idiopathic infertility: does the immunization-dependent Th2 cytokine overbalance really matter? Arch Immunol Ther Exp (Warsz) 2012;60:151-60.
Cite this article as: Chen J, Liu B, Zhang Y, Ao L, Li Z, Qu B, Li X, Yang J. Effect of immunotherapy on patients with unexplained recurrent spontaneous abortion. Ann Palliat Med 2020;9(5):2545-2550. doi: 10.21037/apm-19-440b 